\title{
Analytic Studies of Colloid Transport in Fractured Porous Media
}

\author{
Y. Hwang, P. L. Chambré, W. W.-L. Lee, and T. H. Pigford \\ Department of Nuclear Engineering \\ University of California
}

and

Earth Sciences Division

Lawrence Berkeley Laboratory

University of California

Berkeley, California 94720

November 1989

This work was supported by the Dircctor, Office of Civilian Radioactive Waste Management, Office of Systems Integration and Regulations, Licensing and Compliance Dy/fifgefthe $5 \mathrm{~S}$. Department of Energy under Contract No. DE-AC03-76SF00098. 


\title{
Analytic Studles of Colloid Transport in Frectured Porous Medla
}

\author{
Y. Ewang, P. L. Chnmbre, W. W.-L. Lee, and T. H. Pigford \\ Department of Nuclear Engineering, University of Calfornia \\ and
}

Lawrence Berkeley Laboratory, Univenity of Calfornin, Berkeley, CA 94720

\section{Introduction}

We analyze the interactive migration of radioactive colloids and solute in fractured rock. Two possible interactions between radionuclides as colloids and as solute are considered: (1) solute sorption on nonradioactive colloids to form pseudocolloids, and (2) dissolution of radioactive colloids.

Previous studies ${ }^{1,2}$ have discussed the formation and transport of colloids in porous media, including removal of colloids by filtration and sedimentation. Colloids can migrate faster than solute because of weaker sorption on stationary solids and because of hydrochromatography of colloid particles in flow channels. However, the migration of colloids and pseudocolloids can be retarded by the interaction of colloids with solute, and the migration of solute in local equilibrium with colloids can be more rapid than if colloids were not present. Here we present a new quantative analysis to predict the interactive migration of colloids and solute in porous and fractured media.

\section{Pseudo-collaid migration}

Consider a radioactive solute at concentration $C_{2}(x, t)$ in water in the fracture of fractu: ed porous rock. Also present in the fracture are natural colloids, on which solute can sorb to concentration $C_{1}(x, t)$ on the colloids to form pseudocolloids. We assume the one-dimensional convective-diffusive transport within the fracture, and assume that colloids are too large to diffuse into the rock matrix. Neglecting possible colloid filtration within the fracture, the equation governing the transport of solute as pseudocolloids is:

$$
\epsilon_{1} \xi_{1} \frac{\partial C_{1}(x, t)}{\partial t}+\epsilon_{1} \xi_{1} v_{1} \frac{\partial C_{1}(x, t)}{\partial x}+\epsilon_{1} S_{1}(x, t)+\epsilon_{1} S_{2}(x, t)-\epsilon_{1} \xi_{1} D_{1} \frac{\partial^{2} C_{1}(x, t)}{\partial x^{2}}+\epsilon_{1} \xi_{1} \lambda C_{1}=0, \quad x>0, t>0
$$

where $C_{1}(x, t)$ is the amount of species sorbed on the colloid per unit volume of solid colloid, $v_{1}$ is the colloid pore velocity, $D_{1}$ is the colloid dispersion coefficient, $\lambda$ is the decay constant, $\epsilon_{1}$ is the porosity within the fracture, $\xi_{1}$ is the constant volume fraction of colloids in fracture liquid, $\epsilon_{1} S_{1}(x, t)$ is the rate of sorption to stationary solid, and $\epsilon_{1} S_{2}(x, t)$ is the rate of desorption from the pseudocolloid.

For the same species as solute in liquid in the fracture

$$
\epsilon_{1} \frac{\partial C_{2}(x, t)}{\partial t}+\epsilon_{1} v_{2} \frac{\partial C_{2}(x, t)}{\partial x}-\epsilon_{1} S_{2}(x, t)+\epsilon_{1} S_{3}(x, t)-\epsilon_{1} D_{2} \frac{\partial^{2} C_{2}(x, t)}{\partial x^{2}}+\epsilon_{1} \lambda C_{2}+\frac{q(x, t)}{b}=0, \quad x>0, t>0
$$

where $C_{2}(x, t)$ is the solute concentration in the fracture liquid, $v_{2}$ is the solute pore velocity, $\epsilon_{1} S_{3}(x, t)$ is the rate of solute sorption on stationary fracture solids, $b$ is the fracture half-vidth, and $q(x, t)$ is the diffusive solute flux into the rock matrix, given by

$$
q(x, t)=-\left.\epsilon_{p} D_{p} \frac{\partial N(x, y, t)}{\partial y}\right|_{y=b}, \quad x>0, \quad t>0
$$

$\epsilon_{p}$ is the rock porosity, $D_{p}$ is the solute diffusion coefficient in water in porous rock, and $N(x, y, t)$ is the solute concentration in pore water in the rock.

For solute species sorbed on stationary fracture solids

$$
\left(1-\epsilon_{1}\right) \frac{\partial C_{3}(x, t)}{\partial t}-\epsilon_{1} S_{3}(x, t)+\left(1-\epsilon_{1}\right) \lambda C_{3}(x, t)=0, \quad x>0, \quad t>0
$$


where $C_{3}(x, t)$ is the concentration of sorbed solute species..

For species sorbed as pseudocolloids on the stationary fracture solids

$$
\left(1-\epsilon_{1}\right) \xi_{2} \frac{\partial C_{1}(x, t)}{\partial t}-\epsilon_{1} S_{1}(x, t)+\left(1-\epsilon_{1}\right) \xi_{2} \lambda C_{1}(x, t)=0, \quad x>0, \quad t>0
$$

where $\xi_{2}$ is the constant volume fraction of the sorbed colloid per unit volume of the stationary fracture solid.

Inside the rock matrix

$$
R_{p} \frac{\partial N(x, t)}{\partial t}-D_{p} \frac{\partial^{2} N(x, t)}{\partial y^{2}}+R_{p} \lambda N(x, t)=0, \quad x>0, \quad t>0, \quad y>0
$$

where $R_{p}$ is the solute retardation coefficient in the rock matrix.

We assume linear sorption equilibrium between the solute species in the fracture liquid and the same species sorbed on the colloid. Both the solute species and the colloids in the fracture liquid are assumed to undergo linear sorption equilibrium with the fracture solids

$$
K_{d_{1}}=\frac{\xi_{2}}{\xi_{1}}, \quad K_{d_{2}}=\frac{C_{3}(x, t)}{C_{2}(x, t)}, \quad K_{d_{3}}=\frac{C_{1}(x, t)}{C_{2}(x, t)}
$$

Adding eq. (1)-(5) with equation (7), we can obtain the equation for $C_{1}$ in terms of the effective retardation factor $R$, dispersion coefficient $D$, and velocity $v$.

$$
R \frac{\partial C_{1}(x, t)}{\partial t}+v \frac{\partial C_{1}(x, t)}{\partial x}-D \frac{\partial^{2} C_{1}(x, t)}{\partial x^{2}}+R \lambda C_{1}(x, t)+\frac{q(x, t)}{\epsilon_{1} b}=0, \quad x>0, \quad t>0
$$

where

$$
\begin{gathered}
R=\left[\xi_{1}\left(1+\frac{1-\epsilon_{1}}{\epsilon_{1}} K_{d_{1}}\right)\right]+\left[1+\frac{1-\epsilon_{1}}{\epsilon_{1}} K_{d_{2}}\right] \frac{1}{K_{d_{3}}} \\
v=v_{1}\left(\xi_{1}+\frac{v_{2}}{K_{d_{3} v_{1}}}\right) \\
D=D_{1}\left(\xi_{1}+\frac{D_{2}}{D_{1} K_{d_{3}}}\right)
\end{gathered}
$$

Then the solute and pseudocolloid apparent migration speed is

$$
\frac{v}{R}=\frac{v_{1}\left(\xi_{1}+\frac{v_{2}}{K_{d_{3} v_{1}}}\right)}{\left[\xi_{1}\left(1+\frac{1-\varepsilon_{1}}{\epsilon_{1}} K_{d_{1}}\right)\right]+\left[1+\frac{1-\varepsilon_{1}}{\epsilon_{1}} K_{d_{2}}\right] \frac{1}{K_{d_{3}}}}
$$

Depending on parameters, the apparent speed can be greater or less than the apparent speed of solute without colloid-solute sorption interaction.

The initial and boundary conditions are

$$
\begin{aligned}
N(x, \infty, t) & =0, \quad x>0, \quad t>0 \\
N(x, b, t) & =\frac{1}{K_{d_{s}}} C_{1}(x, t), \quad x>0, \quad t>0 \\
N(x, y, 0) & =0, \quad x>0, \quad y>0 \\
C_{1}(0, t) & =K_{d_{3}} C_{0}, \quad t>0 \\
C_{1}(\infty, t) & =0, \quad t>0 \\
C_{1}(x, 0) & =0, \quad \quad x>0
\end{aligned}
$$


where $C_{o}$ is the inlet solute concentration.

The solution is

$$
\begin{gathered}
C_{1}=\frac{2 K_{d_{s}} C_{o}}{\sqrt{\pi}} \int_{\frac{5}{3} \sqrt{R / D t}}^{\infty} \exp \left\{-\frac{\sqrt{v^{2}+4 R \lambda D}-v}{2 D} x\right\} \operatorname{erfc}\left[\frac{x^{2} \epsilon_{p} \sqrt{D_{p}} R_{p}}{8 D b \epsilon_{1} \eta^{2} \sqrt{t-\frac{R x^{2}}{4 D \eta^{2}}}}\right] \exp \left[-\left(\eta-\frac{x v}{4 D \eta}\right)^{2}\right] d \eta \\
x \geq 0, t \geq 0
\end{gathered}
$$

Figure 2 illustrates the predicted concentrations of solute and pseudocolloids as a function of distance from the fracture inlet, assuming a step-function source of pseudocolloids and solute at the relative concentrations shown at the fracture inlet. In figure 3 the solute concentration is shown as a function of distance from the fracture inlet for various $R$ in the equation (9) where $R$ has the distribution coefficients between solute and colloid, etc. Here the solute-colloid distribution coefficient is held constant. As we expect the front migrates faster for smaller $\mathrm{R}$.

\section{True colloid migration}

Consider a radioactive solute and colloids of the same species in fracture of fractured porous rock. We assume one-dimensional advective transport of colloids and solute in the fracture. Here we assume that the rock surrounding the fracture is impermeable against solute and colloids. Therefore, the equations will also apply to a porous medium. Considering colloid filtration within the fracture, the colloid transport governing equation is:

$$
\begin{gathered}
\epsilon_{1} \frac{\partial C_{1}(x, t)}{\partial t}+\epsilon_{1} v_{1} \frac{\partial C_{1}(x, t)}{\partial x}+\epsilon_{1} S_{1}(x, t)+\epsilon_{1} S_{2}(x, t)=0, \\
x>0, \quad t>0
\end{gathered}
$$

where $C_{1}(x, t)$ is the true colloid concentration in liquid and $v_{1}$ is the true colloid pore velocity, and the other terms are the same as defined in equation (1).

Similar to equation (2) for the solute in the liquid

$$
\epsilon_{1} \frac{\partial C_{2}(x, t)}{\partial t}+\epsilon_{1} v_{2} \frac{\partial C_{2}(x, t)}{\partial x}+\epsilon_{1} S_{2}(x, t)+\epsilon_{1} S_{3}(x, t)=0, \quad x>0, \quad t>0
$$

For solute sorbed on the rock

$$
\left(1-\epsilon_{1}\right) \frac{\partial C_{3}(x, t)}{\partial t}-\epsilon_{1} S_{3}(x, t)+\left(1-\epsilon_{1}\right) \lambda C_{3}(x, t)=0, \quad x>0, \quad t>0
$$

where $C_{3}(x, t)$ is the concentration of sorbed solute on the rock

For colloids sorbed on the rock

$$
\left(1-\epsilon_{1}\right) \frac{\partial C_{4}(x, t)}{\partial t}-\epsilon_{1} S_{1}(x, t)=0, \quad x>0, \quad t>0
$$

where $C_{4}(x, t)$ is the sorbed colloid concentration per unit solid volume

Assuming linear sorption equilibrium between the species in the liquid and on stationary solid the retardation coefficients are

$$
\begin{aligned}
& R_{1}=1+\frac{1-\epsilon_{1}}{\epsilon_{1}} \frac{C_{4}(x, t)}{C_{1}(x, t)} \\
& R_{2}=1+\frac{1-\epsilon_{1}}{\epsilon_{1}} \frac{C_{3}(x, t)}{C_{2}(x, t)}
\end{aligned}
$$

I sing the linear sorption assumption, we have

$$
R_{1} \frac{\partial C_{1}(\boldsymbol{r}, t)}{\partial t}+t_{1} \frac{\partial C_{1}(\boldsymbol{x}, t)}{\partial \boldsymbol{x}}+S_{2}(x, t)=0, \quad x>0, \quad t>0
$$




$$
R_{2} \frac{\partial C_{2}(x, t)}{\partial t}+v_{2} \frac{\partial C_{2}(x, t)}{\partial x}-S_{2}(x, t)=0, \quad x>0, \quad t>0
$$

We now have the three unknowns $C_{1}(x, t), C_{2}(x, t)$, and $S_{2}(x, t)$ but only two equations.

Where colloids are present, we expect that the solute will be at saturation concentration. This suggests the assumption

$$
C_{2}(x, t)=C, U\left(t-\frac{\alpha x}{v_{2}}\right), \quad x \geq 0, \quad t \geq 0
$$

where $C$, is the solute solubility limit and $\frac{v_{2}}{\alpha}$ is the effective migration velocity controlled by colloid dissolution, with $\alpha$ yet to be determined.

Adding equation (26) to (27), substituting equation (28) and neglecting decay.

$$
R_{1} \frac{\partial C_{1}(x, t)}{\partial t}+v_{1} \frac{\partial C_{1}(x, t)}{\partial x}+\left(R_{2}-\alpha\right) C, \delta\left(t-\frac{\alpha x}{v_{2}}\right)=0, \quad x>0, \quad t>0
$$

We solve the above equation using the method of characteristic or of extended Laplace transform applicable to a generalized function with the following side conditions

$$
\begin{array}{rlrl}
C_{1}(x, 0) & =0, & t>0 \\
C_{1}(0, t)=C_{0}, & x>0
\end{array}
$$

where $C_{0}$ is the inlet colloid concentration. Then the solution is

$$
C_{1}(x, t)=C_{0} U\left(t-\frac{x R_{1}}{v_{1}}\right)+\frac{\left(R_{2}-\alpha\right) v_{2}}{\alpha v_{1}-R_{1} v_{2}} C_{0}\left[U\left(t-\frac{\alpha x}{v_{2}}\right)-U\left(t-\frac{R_{1} x}{v_{1}}\right)\right], \quad x \geq 0, \quad t \geq 0
$$

To specify the yet-unknown quantity $\alpha$, assume the colloid and the solute migration fronts move at the same velocity $\frac{v_{2}}{\alpha}$ in the fracture.

Therefore,

$$
C_{0}-\frac{\left(R_{2}-\alpha\right) v_{2}}{\alpha v_{1}-R_{1} v_{2}} C_{9}=0
$$

Therefore, the colloid concentration is

$$
C_{1}(x, t)=C_{0} U\left(t-\frac{\alpha x}{v_{2}}\right), \quad x \geq 0, \quad t \geq 0
$$

where

$$
\alpha=\frac{\left(R_{1} C_{0}+R_{2} C_{1}\right) v_{2}}{C_{0} v_{1}+C_{,} v_{2}}
$$

Pliysically the colloid and solute migration front with dissolution is between the colloid migration front and the solute migration front without dissolution. Therefore, a satisfies

$$
\frac{v_{1}}{R_{1}}<\frac{v_{2}}{\alpha}<\frac{v_{2}}{R_{1}}
$$

Figure 3 presents profiles of colloid and solute concentrations for the specified data set. Without colloid dissolution, at a thousand years since radionuclides are released into the fracture inlet, the colloid migration front locates 100 meters from the inlet and t: ${ }^{\circ}$ solute migration front is 20 meters from the inlet. But witl colloid dissolution into solute, the colloid front is at 800 meters away from the inlet and the solute front also advances to the same 800 meters. Therefore, the dissolution mechanism is important to the solute migration in the fracture, especially when the inlet colloid concentration is much greater than that of the solute. This can happen when oxidized actinides suddenly meet a redox front, precipitate, and form colloids. 
Then the solute concentration will be controlled by the reduced solubility limit, but a colloidal aggregate of the precipitated solute can be at much higher concentration.

This analysis is a tool to investigate the assumption of colloid dissolution. Experimentally $v_{1}, v_{2}, R_{1}$, and $R_{2}$ are measurable, and based on the colloid dissolution assumption, $\frac{v_{2}}{\alpha}$ is also measurable. Agreement of the measured $\alpha$ with the theoretical value from equation (35) would validate the colloid dissolution assumption.

\section{Conclusions}

The migration of colloids and solute in fractured medium with two types of colloid-solute interaction has been studied. When the colloid-solute interaction is by dissolution, solute that normally has greater retardation than colloids is accelerated by colloid dissolutions. For sorption interaction, the apparent migration speed of pseudocolloids can be greater or less than the solute migration speed without interaction, depending on the chice of parameters. Further studies of the interactive migration of colloids and solute with dissolution equilibria are under way.

\section{References}

1. A. Avogadro and G. De Marsily, "The Role of Colloids in Nuclear Waste Disposal " Material Research Society, vol 26, pp 495-505, 1984

2. B.J. Travis and H.E. Nuttall, "A Transport Code for Radiocolloid Migration: With an Assessment of an Actual Low-Level Waste Site," Material Research Society, vol 44, pp 969-976, 1985. 


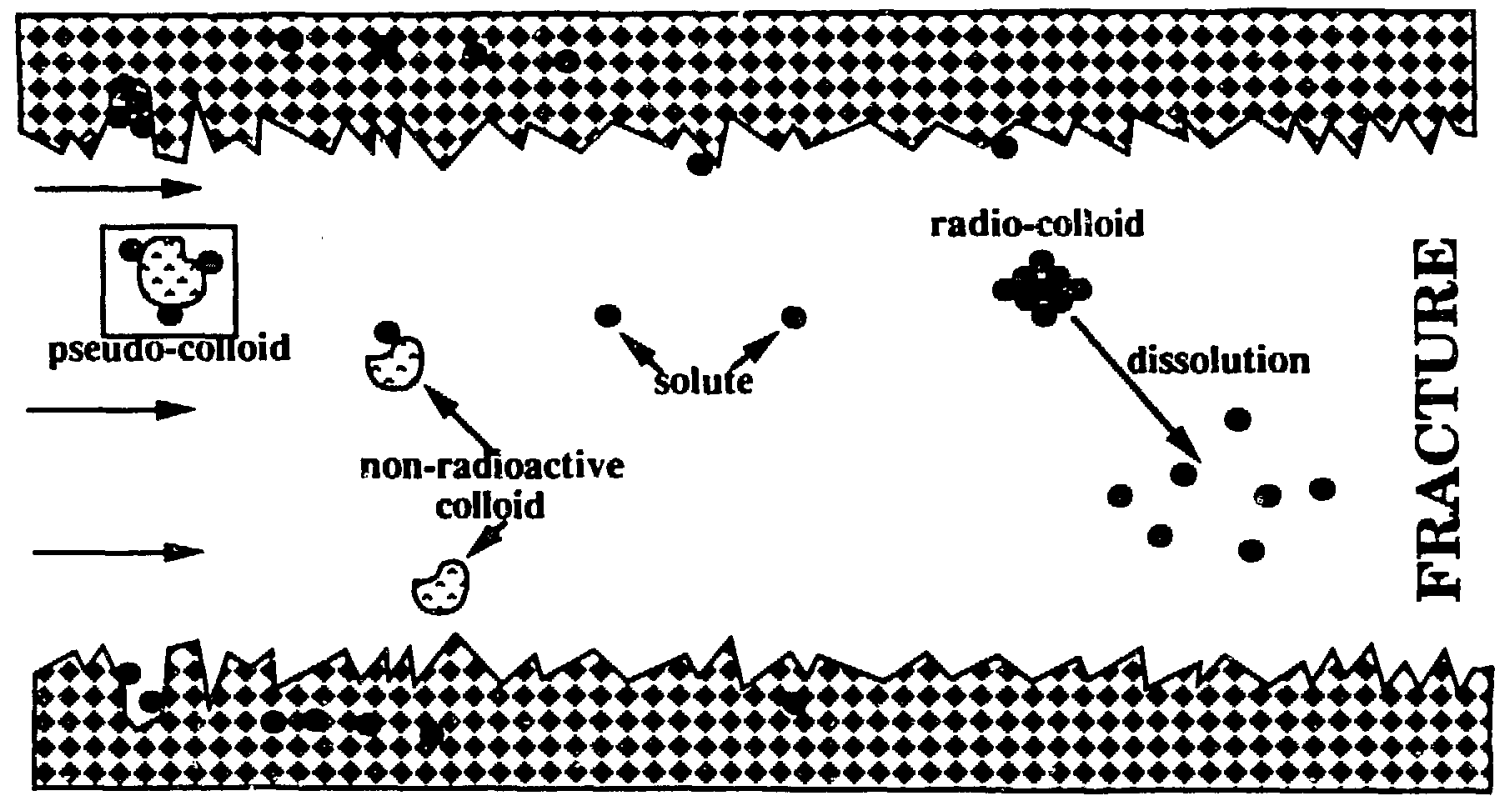

Figure 1. Colloid Migration in a Fracture

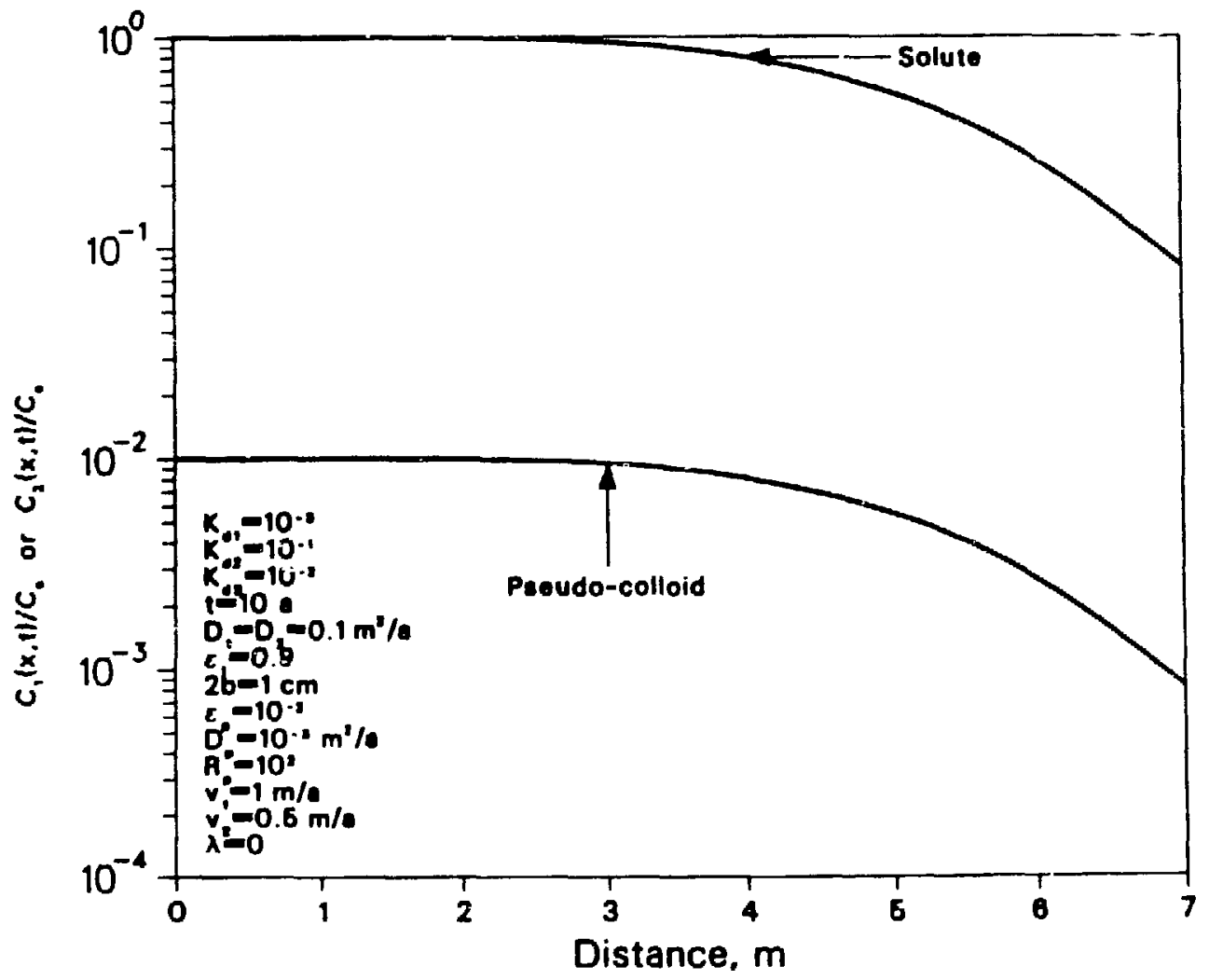

Figure 2. Aelative Concentration of Pseudocolloid and Solute with Fracture Flow and Metrix Diffueion of Solute 


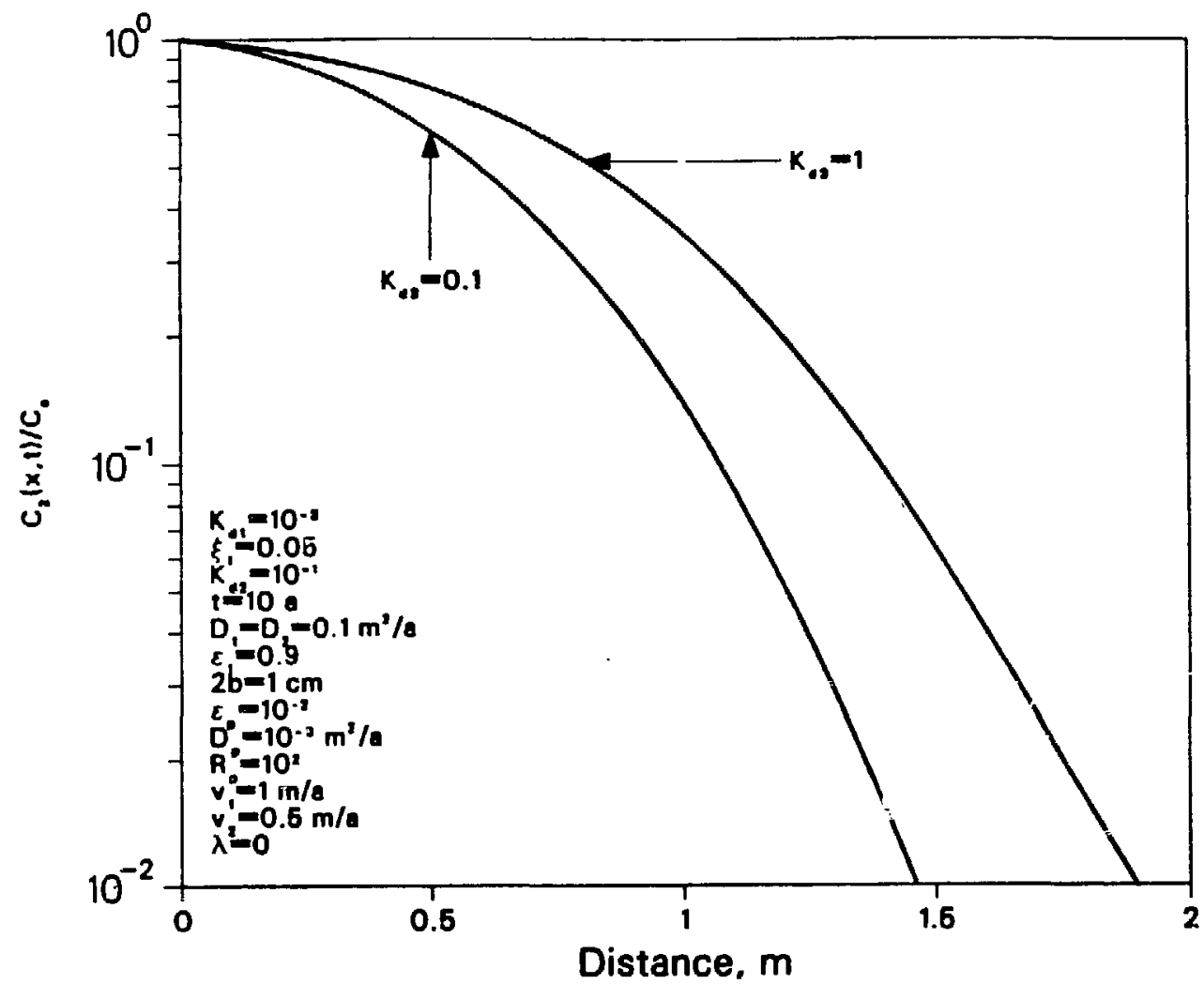
Figure 3. Relative Concentration of Solute for Different $K$ do
with Frecture Flow and Solute Matrix Diffusion

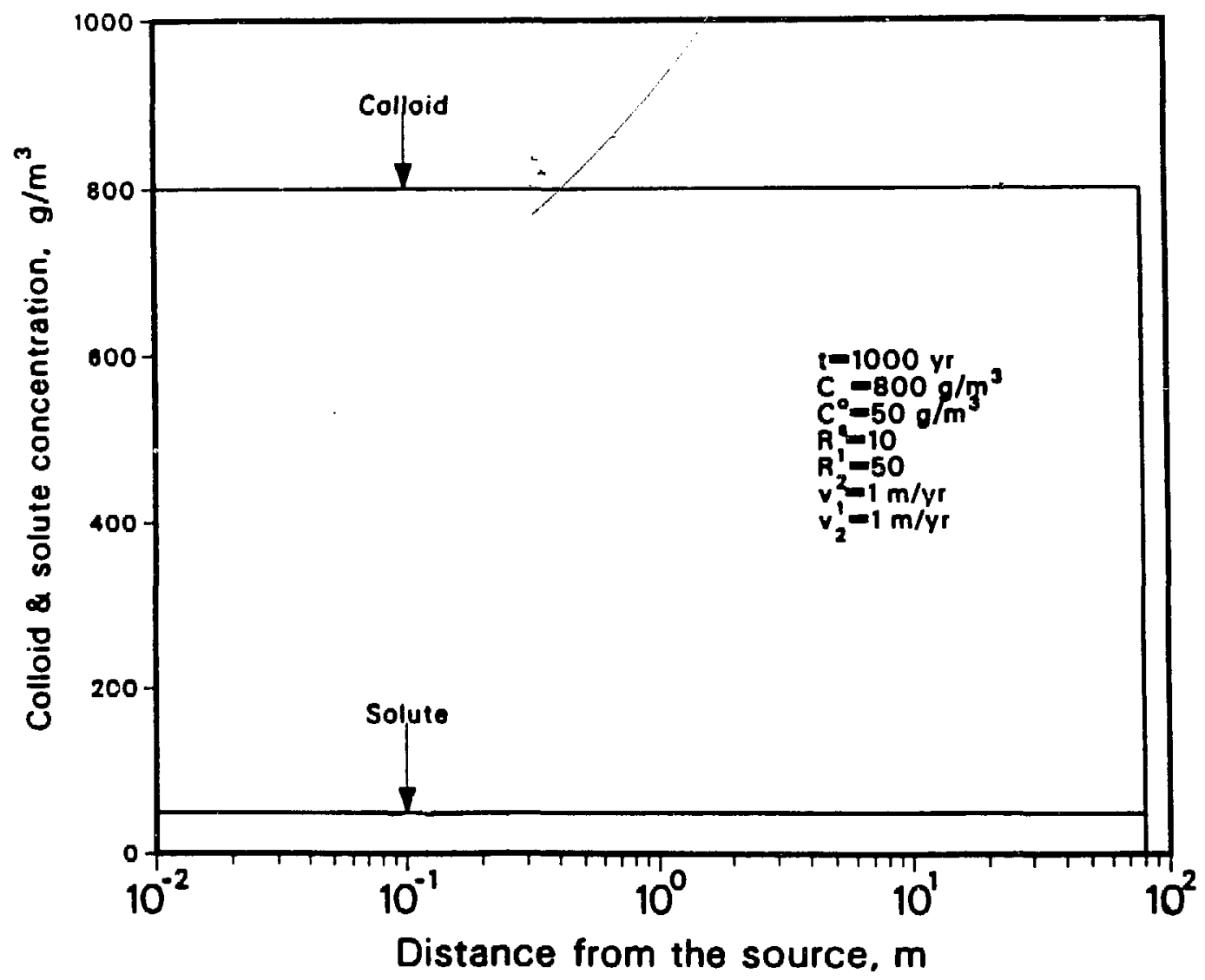

Figure 4. Concentration of Colloid and Solute in the Fracture with Colloid Dissolution to Solute 